ber in rinigen Zropfen verbinnter Salpetersäure aufgenoms men burch falpeteriaures Cilber merplí getríbt wurde.

i) Die lesten Risrnden, welde mit ellolid nod librig waren, bradte id in ein all einem Mishrden geblafenes Tubus

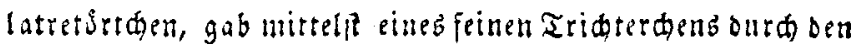
Tubulus einige Tropien Ueşnatronflifingest himein, uns

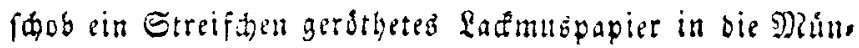

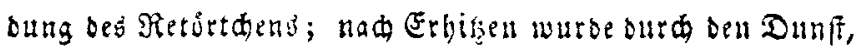

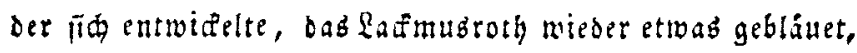
aber fo menig, bав i申 nith befimmt entfdeiben mag, ob bie Soncretion aud eine Itmmoniatyertinbung enthalten

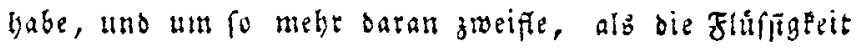

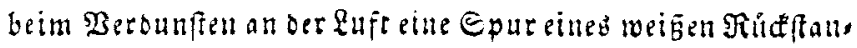

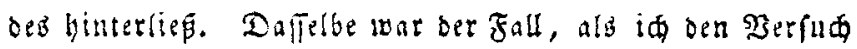
mit cinem fpáter erbaltenen Stifdaen biefer Soncretion swieserholte, weldiz $\frac{\text { I }}{8}$ ङran wog.

k) Die unteriudte Eoncretion befegt fona aus: ploophorfaurem Ralt;

Euglenraurem Ralt;

foblemfaurer Bittererbe;

Edieim;

eimeitâfoffartige Materie;

Eglormatrium Epuren.

\title{
Ebemilate Rotizen;
}

\section{vom}

Profefine Gielger in Seitelberg.

(IIUb́ einem ভøreibell an R. Branbeb).

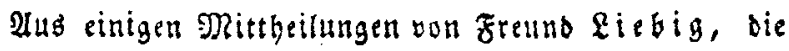
Du im Magajin finden wirft, gegt bervor, baf bas loges

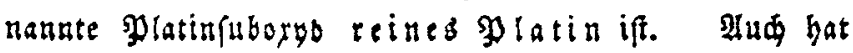


gieblg eine neure Drybationgfuffe seg Cyanz entbect, Untercyanfáre, und dáb bie Gåure aub dem \$ferber harn, seld man fur $\mathfrak{B}$ enjoefäure gebalten bat, Eeine Ben, joefunte iff.

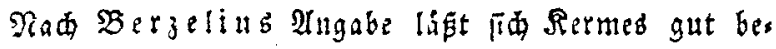
reiten. Die Sdlippefde Rethooe, Goldidwefel jul bes reiten, finte id rehr praftifi, uno jiehe ire jeber andern

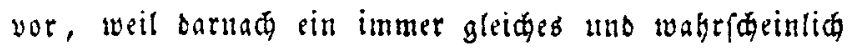
gans reines poraparat erhalten wits. 2lud mittelfi fauten fómefelfautem Rafi, Ediveferantimon und Rale, nad mei, ner frübern Ingabe lafen fid burd frarkes sersampfen ber Eauge Rryftalle erfialten, bie ein gleides Mráparat liefern. Intereffant ift dab abuectaende Nerhalten bes Rermes uno Borbidimefels ju Merc. dulc., wenn fite feudt uno lufts

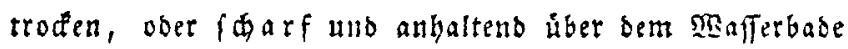
aubgetrodnet, banit gerieben seerben. In beiben erffern Fållen wirb bas bemenge fínell ounpel, unter Bitoung won Squefelqueffilber unb Butyr. antimonii, wie if f(ton vor 20 Jahren beobadtet habe; aber bas fifarf getrodnete

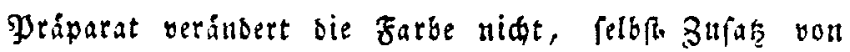
WBaffer bemireft bie gegenfeitige zerjeşung nidt und es ift anthaltenoes Erroirmen bazu nsthig. Deuter biefes nidet

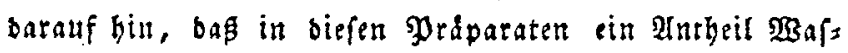
Per innig gebunben (afb Seybratroaffer?), uno nidt blos

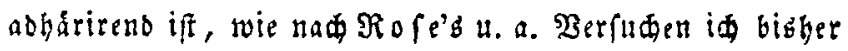

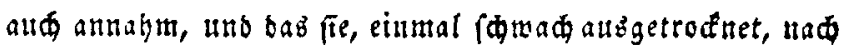
und nad rieber anjiehen? Man erinnere fich hiebei nut an oab analoge $\mathfrak{B e r h a l t e n ~ d e s ~ f r i f ~ g e t r o d n e t e n ~ M l a u n b . ~}$

Sn bem Salmiapgeifte fano id einjeinfades sRitter, Rermez uno bolofámefel zu anterfaciben unb zu prúfen;

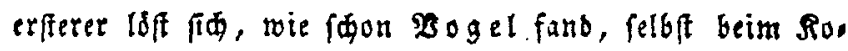
Wen bamit fegr werig auf, lekgterer, bagegen ourd gelinbe

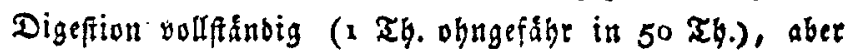


nut weun et 2 SR. G. Shivefel gegen $x$ SנR. G. Intimon enthalt, entgalt er mebr Sdwefel, fo bleibt siefer unauf, gelsft, enthalt er Rermeg, fo bieibt bisfer jurúa.

Beobaditungen liber Die Saure, weldye bas

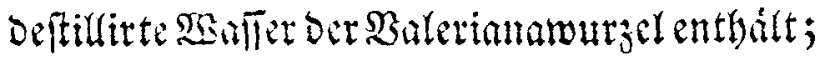

von

S. $\Re$. Sijote,

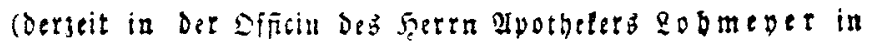
Serber).

Bor einigen Jabren fdon made id bie Bemerfung,

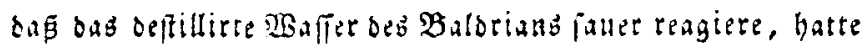
aber nide weiter barauf geadtet, bis id vor einiger zeit Lurd eine Nutij liber biefen Gegenfand von ferrn

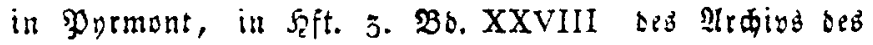
Ipothetervereins wieder darauf aufmerfam gemade ivurde.

Da id gerabe im Selitze einer Suantitat fehr concens


fude bamit alluffellen, "Im zu erfabren, ob meine Bermu, thung, baf bie Säure, yielleidt beim langfamen Trofnen ber Baldrianwurgeln ergeugl, Efipglaure ley, gegriubet fey.

Dab Daffer, weldes iq bazu anwaubte, war bab I8 $\mathfrak{Y}$ funb p. c. betragende Deftillat yon 56 PFuno p. c. bet

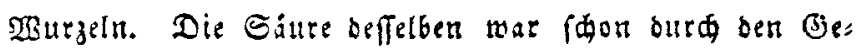
fomat beutlid bemerpbar; i申 fätigte fie mit Eryfallifirs tem Poblenfaurem $\mathfrak{N a t r u m , ~ s o o v o n ~ g e n a u ~} 2$ Unzen uno $j$

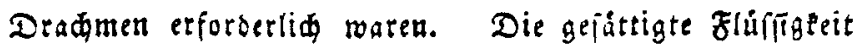
raudte iq bei gelindem Fetter bis zu 6 unjen ab. Beim Iboampfen hatte fi由 ein Theil ber Såure verfúdtigt, benn 